INTERNATIONAL DESIGN CONFERENCE - DESIGN 2018

https://doi.org/10.21278/idc.2018.0479

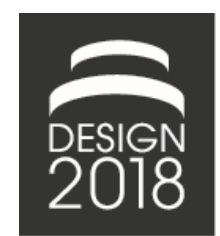

\title{
INSIGHTS INTO DESIGN CONCEPT SIMILARITY JUDGEMENTS
}

\author{
C. P. McTeague, A. Duffy, L. Hay, T. Vuletic, G. Campbell, P. L. Choo and M. Grealy
}

\begin{abstract}
Similarity has been shown to influence various measures of outcome creativity in combinatorial design tasks, but the role of similarity during the combination of design concepts is unknown. As an initial step towards understanding design concept similarity we review prominent models of similarity in psychology and carry out an exploratory experimental investigation of design concept similarity judgements. Results suggest that as per non-design stimuli, similarity may be the result of structural alignment processing and similarity ratings may be related to the degree of common features.
\end{abstract}

Keywords: similarity, conceptual design, design creativity, cognitive capabilities

\section{Introduction}

Similarity is an important construct in many aspects of human behaviour, (Tversky, 1977; Markman and Gentner, 1996). Similarity has also been shown to play a role in various forms of combinatorial design creativity, and may further play a role in perceptions of novelty. During analogical reasoning exposure to surface dissimilar analogies can increase the novelty of generated concepts (Wilson et al., 2010; Chan et al., 2011), and can also result in more variation in quality (Chan et al., 2011). When designers are tasked with combining pairs of objects represented pictorially to create a new design concept, originality is seen to improve with decreasing similarity of base concepts (Nagai et al., 2009), and overall creativity improves when stimuli are from different taxonomic categories but have high relative similarity (Jang, 2014). Further, similarity has been proposed as a component of novelty judgements (Brown, 2016) but this appears to be a relatively unexplored area of research.

There is an opportunity, to expand existing research on similarity in two key areas. Firstly, the previous creative combination studies (Nagai et al., 2009; Jang, 2014) have utilised common, taxonomically organisable objects as stimuli (e.g. car, elevator, scissors). No studies have been found, however, which use sketch representations of conceptual design concepts as stimuli in combination tasks. This is important because the combination of design concepts is prescribed in design methods such as SCAMPER (van Boeijen et al., 2014) and has been identified in protocol studies of conceptual design (Hay et al., 2017). If there is a relationship between pair-similarity and the creativity of outcomes with taxonomically organisable concepts, the same may hold when the stimuli are representations of design concepts. Secondly, although similarity has been identified as a potential component of novelty perception, the lack of research on similarity perception precludes comparison of these phenomena.

In order to reliably manipulate the similarity of design concepts in creativity experiments or investigate the relationship between similarity and novelty in design, it is necessary to understand how designers make similarity judgements and what aspects of design concepts determine similarity. Towards such an understanding, we first report on literature from psychology to gain insights into the cognitive processes and determinants of similarity in non-design concepts. We also review existing similarity metrics in 
design, and carry out an experiment to gain insights into cognitive processing and determinants of similarity in design concepts.

\section{Similarity}

Perceptions of similarity are thought to be the result of two independent cognitive processes described by a dual-process model of similarity (Golonka and Estes, 2009). Feature-based similarity is thought to be the primary process and is a function of the common and different features between two concepts. Thematic similarity is an important secondary process which acts on concepts with pre-existing thematic relations.

\subsection{Feature-based similarity}

Feature-based similarity results from the comparison of the mental representational structures of two concepts. An early feature-based model, Tversky's contrast model (Tversky, 1977) assumed that similarity is a positive function of the common features of a pair of items and a negative function of the different features. A key limitation of this model is that it does not account for perceptions of similarity derived from relations between entities. We provide an example of the importance of concept relations adapted from Gentner and Markman (1995).

a)

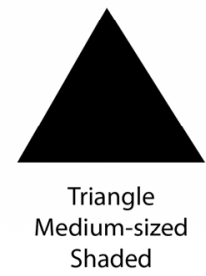

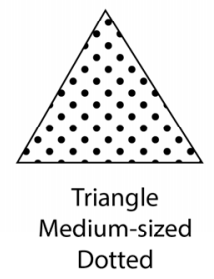

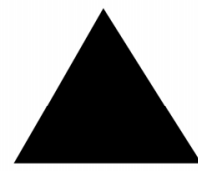

b)

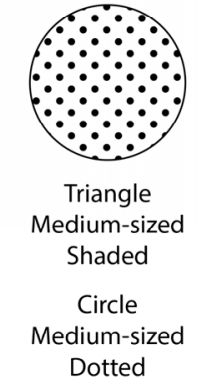

Above
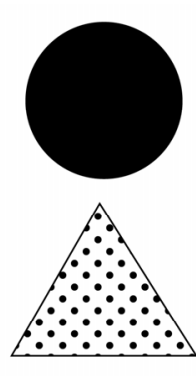

Circle

Medium-sized

Shaded

Triangle

Medium-sized

Dotted

Figure 1. Similarity as intersecting feature sets, adapted from Gentner and Markman (1995)

In this example, 'entity' refers to each individual circle or triangle, and 'set' refers to multiple entities grouped together. If both entities in Figure 1a are described by a list of features, similarity (according to Tversky's model) would be derived from an intersection of the two feature lists. Thus 'triangle' and 'medium-sized' become commonalities, while 'shaded' and 'dotted' are differences. Similarity is then a positive function of the commonalities and a negative function of the differences. In Figure $1 \mathrm{~b}$ however, the introduction of multiple entity's in each set creates additional demands on a model of similarity. When represented as lists of features, the contents of both lists are the same, albeit listed in a different order. If similarity is simply a function of the commonalities and differences between two feature lists, then these sets of objects would be identical. Intuitively however this is not the case; thus raising the need for a cognitive mechanism which decides on which features to compare with which other features. The sets can be aligned in one of two ways: (i) the circles are placed in correspondence, thus geometry is common, but fill and relative location are different, or (ii) the top most shapes are placed in correspondence, thus fill is common, but geometry and relative location are different. Regardless of the outcome, this demonstrates the need for alignment of representations.

The structural alignment model of similarity accounts for the relations between entities by making assumptions about the nature of cognitive representations and the processes which compare those representations. Representations are assumed to consist of object descriptors (attributes), objects 
(entities), functions, and relations between elements (Markman and Gentner, 1996). Taking Figure 1b as an example, the relation in the left set would be described of the form 'above (circle, triangle)', and the entities would in turn have the attributes 'shaded' and 'dotted'.

The structural alignment model also specifies how commonalities and differences contribute to judgements of similarity by distinguishing between two types of differences: alignable differences and nonalignable differences. Similarity is a function of the commonalities, alignable differences and nonalignable differences between the two representational structures of a pair of concepts. In other words, similarity is determined by the alignability of two concepts.

It is not currently known whether the structural alignment model also applies to similarity judgements of design concepts. A key gap in knowledge are how design concepts might be described in terms of structured representations, including attributes, objects and relations.

\subsection{Thematic similarity and thematic relations}

Thematic relations are "the external or complementary relations among objects, events, people, and other entities that co-occur or interact together in space and time" (Lin and Murphy, 2001, p.3). Key features of thematic relations are their externality (occurring between two or more things), and complementarity (fulfilling different roles in the given theme). Estes et al. (2011) claim that thematic relations are derived from affordances or convention. For example, a hammer and nail are said to be thematically related because of their mutual affordances, however a wine glass and dinner plate are thematically related because they conventionally co-occur in a meal theme even though their features do not afford direct interaction. In experimental conditions, participants are seen to justify their similarity ratings of certain concept pairs by thematically relating them (Bassok and Medin, 1997), and pairs of stimuli which are assumed to have pre-existing thematic relations are judged to be more similar than thematically unrelated concepts (Wisniewski and Bassok, 1999).

\section{Integrating similarity research in design and psychology}

A logical starting point would be to determine whether existing models of similarity apply to similarity judgements of design concepts. The type of similarity described by structural alignment theory makes intuitive sense when applied to design concepts. The more features two design concepts share the more similar they would be perceived to be. It is not clear how, if at all, design concepts could be related 'thematically'. Assuming that thematic relations arise from convention or affordance, then thematic similarity may be derived from a perceived sense of potential artefact-artefact affordances (see: Maier and Fadel, 2003). If the dual-process model of similarity applies to judgements of design-concept similarity, then any study of design concept similarity must make provision for the involvement of both types of similarity processing.

Towards understanding the cognitive processing and determinants of design concept similarity judgements, it may sufficient to conduct a grounded investigation into similarity by giving participants random pairs of concepts and evaluating their responses. In psychological studies of similarity however a number of methods have been utilised grant a degree of a priori control over the similarity of input stimuli, ensuring either a range of similarities or distinct high and low similarity groups. Knowledge of how this is achieved would grant further insights into the aspects of design concepts which contribute to perceptions of similarity.

\subsection{Similarity metrics in design research}

In design research, a number of researchers have defined similarity metrics. McAdams and Wood (2002) developed a quantitative metric which computes the similarity of analogous products based on functional similarity and consumer needs. Fu et al. (2013) utilise latent semantic analysis to determine the semantic similarity of patent documents. Bao et al. (2016) propose a metric which measures the unique features of two concepts; the more unique features between a pair, the lower the similarity. By defining similarity in terms of common features, the authors have inherently adopted a structural alignment view of similarity. However, the features are uniquely associated with the design task used are thus not generalisable. 
None of these similarity metrics are supported by human perceptions of similarity. In addition, none of the metrics appear to be applicable to early stage design concepts represented by sketches. Crucially however, none of these metrics aligns with the structured representations assumed by the structural alignment model or accounts for the external, complementary (thematic) relations between concepts.

\subsection{Stimuli creation methods in psychology}

Markman and Gentner (1993) and Markman and Wisniewski (1997) manipulate similarity by pairing concepts from hierarchical concept taxonomies (example Figure 2). Markman and Gentner (1996) systematically manipulate alignability by altering the objects and relations within triads of pictorial scenes. For use in a study of conceptual combination and emergence, Wilkenfeld and Ward (2001) created 90 pairs of concepts assumed to be similar and dissimilar and use external judges to refine the set to the 8 most and least similar. Translation of these methods to design raises a number of issues.

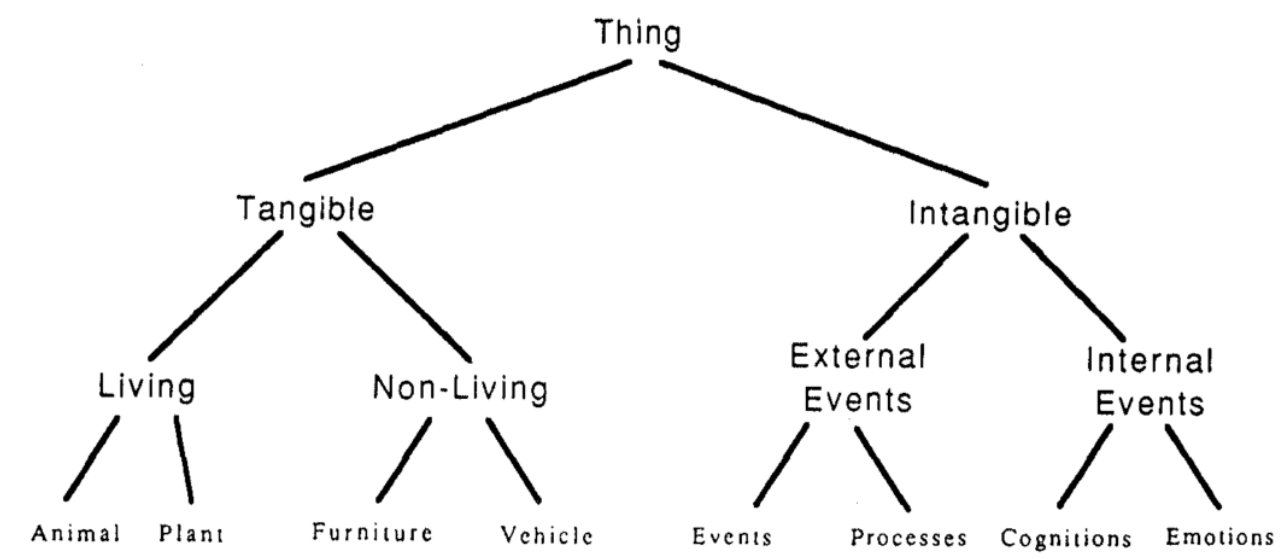

Figure 2. Example of a taxonomic concept hierarchy (Markman and Gentner, 1993)

Hierarchically organised concepts from the same category tend to be more similar than those from other categories because they share common properties based on their superordinate category (Markman and Wisniewski, 1997). This has beenS used to make assumptions about the degree of alignability between pairs, and by extension their similarity. It has been suggested however, (Taura, 2016, p.61), that design concepts cross taxonomic categories and do not share the same conceptual structure. Thus, while taxonomic category location can be used to vary the alignability of everyday concepts a priori, it is unclear as to whether the same holds true for design concepts. Systematic manipulation of the properties and relations of design concepts as per (Markman and Gentner, 1996) would require the artificial creation of stimuli. This inherently precludes the use of genuine concepts created in the design process. The final method, the experimenter intuition and refinement by judges method used by Wilkenfeld and Ward (2001), does nothing to account for potential thematic relations between concepts. Since the potential involvement of thematic processing in design concept similarity is yet unknown, use of this method would be unreliable.

It can be seen that although similarity metrics have been proposed in design, none are generalisable and applicable to early stage design concepts. Further, despite a number of methods for manipulating the similarity of concepts in psychology studies, none offer a reliable means of translation to a design context.

\subsection{Summary of literature insights into design concept similarity judgements}

Towards understanding the cognitive processing of design concept similarity judgements, we sought to gain insights into two key areas. That is, (i) the aspects of design concepts which contribute to perceptions of similarity, and (ii) the types of cognitive processing involved in similarity judgements. Structural alignment processing predominates in non-design similarity judgements, and in such cases similarity is determined by the degree of alignability (common features) between two concepts. If the same form of processing predominates in similarity judgements of design concepts, it would be expected 
that alignability should vary with similarity. By varying the degree of alignment of design concept features and eliciting similarity judgements, it should be possible to evaluate the degree to which those features are drivers of similarity in design concepts. Since there does not appear to be a generalisable metric for similarity in design, we can attempt to manipulate abstract artefact variables such as function or means. To address the type of cognitive processing involved, we can also elicit explanations from participants about the reasons for their similarity judgements and analyse them for evidence of featural or thematic processing.

\section{Similarity experiment}

To gain insights into cognitive processing and the design concept features which drive similarity, we carried out an experiment with two key aims:

Aim 1. To test the relationship between concept pair alignability and participants judgements of similarity

Aim 2. To determine which types of cognitive processing are used by designers to arrive at judgements of similarity

We explored the possibility that design concepts may be hierarchically organisable and created pairs of concepts which varied in terms of alignability. We achieved the first aim via similarity ratings of design concepts and the second by eliciting explanations for similarity ratings. The following assumptions were made:

1. A solution space of design concepts can be organised into an unambiguous hierarchical structure

2. Design concepts can be described in terms of the Need $[\mathrm{N}]$ and sub-need [Ns] which they address, their most salient (purpose) function [F], and the means of fulfilling that function [M]

3. Concept pair alignability can be varied by manipulating the number of common N, Ns, F and M variables

4. If structural alignment processing is involved to some degree, then similarity explanations will contain evidence of featural commonalities or differences

5. If thematic processing is involved to some degree, the similarity explanations will contain evidence of external, complementary relations between concepts

If structural alignment processing predominates for similarity judgements of design concepts, then we would expect alignability to vary with mean similarity ratings and for explanations of similarity ratings to contain predominantly featural responses.

\subsection{Stimuli creation}

Design concepts were sorted into hierarchical taxonomies by the lead researcher; concepts were then coded in terms of their Ns, N, F and M variables. Four hierarchical taxonomies were created by using concepts generated by different participants in a concept generation task. Concepts generated in response to four design tasks from 36 participants were used for the hierarchical organisation. Table 1 shows each of the four design tasks, the total number of concepts used as input to the categorisation, the number of concepts which did not belong to a clear category (had ambiguous issue, function or means properties), and those which were excluded.

Method. Design concepts were first grouped by the need which they were interpreted to satisfy and then divided into sub-groups according to the sub-need which they addressed, their function and means (see Table 2). This resulted in a maximum of four hierarchical layers, however in some instances there was no need to divide an need into a sub-need. Concepts were excluded if they met any of the following exclusion criteria (i) not a consumer product, i.e. they were wholly an infrastructure or service solution, (ii) did not address the brief, (iii) the designer identified a serious flaw in their own concept, (iv) the concept did not have both a sketch and accompanying annotation, (v) the sketch representation could not be deciphered, or (vi) the means or function of the product could not be interpreted. Concepts were classified as 'ambiguous' if they did not cross the exclusion criteria but there were multiple categories into which they could be placed at any level (see Section 6.3 for discussion on ambiguity and hierarchical organisation). This resulted in a range of concepts grouped under a hierarchical need, function and means structure (see Section 6.3.1 for discussion). 
Table 1. Design tasks used to create the initial design concepts, 'Amb' = ambiguous,

$$
\text { 'Exc' }=\text { excluded }
$$

\begin{tabular}{|c|c|c|c|c|c|}
\hline $\begin{array}{l}\# \\
\begin{array}{l}\# \\
\tilde{D} \\
\tilde{\sigma}\end{array}\end{array}$ & Description & $\stackrel{0}{0}$ & $\begin{array}{l}\text { D } \\
\text { ص }\end{array}$ & 妾 & $\begin{array}{r}\text { u } \\
\text { x }\end{array}$ \\
\hline 1 & $\begin{array}{l}\text { Domestic food waste is a serious problem due to global food shortages and } \\
\text { socio-economic imbalances. Generate concepts for products that may reduce } \\
\text { unnecessary food wastage in the home. }\end{array}$ & 86 & 61 & 16 & 9 \\
\hline 2 & $\begin{array}{l}\text { Camping is a popular activity but can have negative environmental impacts } \\
\text { through disruption to wildlife; litter and pollution of water sources. Generate } \\
\text { concepts for products that reduce the negative impacts of camping. }\end{array}$ & 75 & 47 & 8 & 20 \\
\hline 3 & $\begin{array}{l}\text { Long distance water transportation may be necessary in drought-prone } \\
\text { developing nations but can be problematic due to lack of resources and } \\
\text { infrastructure. Generate concepts for products that may facilitate water } \\
\text { transportation in developing nations. }\end{array}$ & 73 & 38 & 1 & 34 \\
\hline 4 & $\begin{array}{l}\text { Sitting in the same position for long periods may be harmful to health. } \\
\text { Generate concepts for products that may facilitate physical exercise whilst } \\
\text { completing activities in a seated position in the home and office. }\end{array}$ & 85 & 56 & 7 & 12 \\
\hline
\end{tabular}

Table 2. Description of need, sub-need, function and means variables

\begin{tabular}{|l|l|}
\hline Variable & Description \\
\hline Need & $\begin{array}{l}\text { The maximum level of differentiation in the solution space which describes 'how' the concept } \\
\text { addresses the brief. }\end{array}$ \\
\hline Sub-need & A sub-division of need \\
\hline Function & The purpose of the artefact \\
\hline Means & A general term which encompasses artefact behaviour and structure \\
\hline
\end{tabular}

Pairs of design concept sketches were created by selecting concepts according to five conditions (Table 3 ). In condition one, design concepts were common in terms of N, Ns, F and M; in condition five they shared no commonalities across these four variables. A total of 40 pairs of concepts were created: 2 (pairs) x 5(conditions) x 4(design tasks). The pairs were picked from across the hierarchies and were assumed to be equivalent within condition across hierarchies. An example of a pair of concepts from design task 2, condition 4 is presented below (Figure 3). The concepts are interpreted to address a common need, but are different at the sub-need, function and means level.
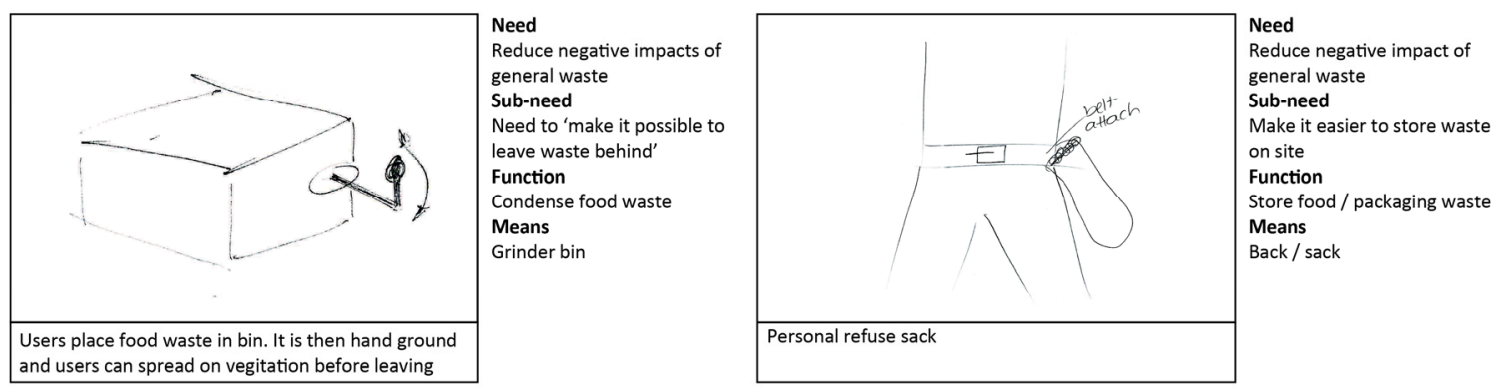

Figure 3. Example of a pair of concepts, design task 2, condition 4

\subsection{Similarity judgements and explanations}

In the similarity rating experiment participants are asked to rate the similarity of pairs of design concepts and explain their rating. The explanations were coded based on the type of cognitive processing evident. 
Participants. 11 individuals, eight with at least three years formal product design engineering education, two with master's degrees in mechanical engineering and 1 psychology $\mathrm{PhD}$ researcher with two years of experience in product design research. All participants were naïve to the aims of the experiment.

Stimuli. 40 pairs of design concept sketches and annotations, eight from each of the five conditions. Each participant received the same set of stimuli in a random order.

Materials. The pairs were printed on A4 landscape paper, with the original design task typed above each pair. Annotations were left on the sketches and additional descriptions provided by the original participant re-typed below the corresponding design concept.

Procedure. Each participant was sat at a computer which prompted them to enter their similarity ratings and explanations. The similarity scale ranged from 1 (not at all similar) to 9 (extremely similar). Participants were instructed to provide similarity ratings by judging the similarity of the design concepts while ignoring the sketch properties of the representations. No time limit was placed on the task.

Design. There were 5 levels of alignability, the dependent variable was the numerical similarity rating.

Table 3. Experimental conditions $(C=$ common, $D=$ different $)$

\begin{tabular}{|c|c|c|c|c|c|}
\hline & \multicolumn{4}{|c|}{ Features } \\
\hline & & Need $(\mathrm{N})$ & Sub-Need (Ns) & Function $(\mathrm{F})$ & Means (M) \\
\hline \multirow{5}{*}{ 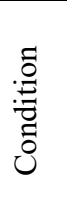 } & 1 & $\mathrm{C}$ & $\mathrm{C}$ & $\mathrm{C}$ & $\mathrm{C}$ \\
\hline & 2 & $\mathrm{C}$ & $\mathrm{C}$ & $\mathrm{C}$ & D \\
\hline & 3 & $\mathrm{C}$ & $\mathrm{C}$ & D & $\mathrm{D}$ \\
\hline & 4 & $\mathrm{C}$ & $\mathrm{D}$ & D & D \\
\hline & 5 & $\mathrm{D}$ & $\mathrm{D}$ & D & $\mathrm{D}$ \\
\hline
\end{tabular}

Scoring. To attempt to determine the means of cognitive processing involved in the participants' similarity judgements, their explanations are coded as 'featural', 'thematic' or 'other' based on definitions from the literature. Featural explanations were expected to contain a commonality, alignable difference or nonalignable difference. Thematic explanations should contain an external, complementary relation between two design concepts. The 'other' code is reserved for explanations which cannot unambiguously be classified by one of the other two codes. The coding was carried out in a single pass by the lead author and was not corroborated with a second rater.

\section{Results}

A preliminary analysis is presented with regards to four areas: the relationship between alignability and similarity, evidence of cognitive processing types, generalisability of findings, and concept features influencing similarity judgements.

\subsection{Relationship between alignability and similarity}

The first aim of the study is to test the relationship between concept pair alignability and participants' judgements of similarity. If similarity varied with the given definition of alignability, then this would suggest that those N, Ns, F and M variables would represent the aspects of design concepts which contribute towards similarity. Table 4 shows the mean similarity ratings for each of the 40 pairs of design concepts. Within each design task and condition there were two pairs (A and B) assumed to be equivalent in terms of alignability.

The rank order of the mean similarity ratings for the condition decrease with alignability in all but two instances (DT6, Pair B, DT9, pair B). This gives the impression of a relationship between alignability and similarity. Although not shown due to space limitations, the standard deviations for the similarity responses appeared high, ranging from 0.40 to 2.32 . Owing to the high variance in many responses, it is premature to attempt to infer significant results from this data, we must first take steps to explore the reasons for such high variability. To this end we continue to address the second aim, namely what type of cognitive processing was used in each similarity rating and then further investigate whether the pairs within each condition are equally good members of that condition. 


\subsection{Type of cognitive similarity processing}

The second aim of the study is to determine which mode of cognitive processing is used by designers to arrive at judgements of similarity. A total of 439 explanations were examined from 440 responses (one similarity rating in the study was not accompanied by an explanation). Of these, 414 were coded as being clearly featural explanations, and 25 as 'other'. Despite the literature definition of thematic relations, no explanations could clearly be identified as thematic. However, of the 25 'other' explanations, six were potentially thematic, but contained insufficient information to decipher the participants intent.

Since no example of thematic processing could be clearly identified, for the purposes of further analysis it is assumed that all similarity judgements were carried out via structural alignment processing. This suggests that the high variance in similarity ratings is not a result of a prevalence of thematic processing.

Table 4. Mean similarity for all pairs of concepts

Mean similarity ratings

\begin{tabular}{|c|c|c|c|c|c|c|c|c|c|c|}
\hline & & \multicolumn{2}{|c|}{ D.Task 1} & \multicolumn{2}{|c|}{ D.Task 2} & \multicolumn{2}{|c|}{ D. Task 3} & \multicolumn{2}{|c|}{ D.Task 4} & \multirow[t]{2}{*}{ Condition mean } \\
\hline & & $\mathrm{A}$ & B & A & B & $\mathrm{A}$ & B & A & B & \\
\hline \multirow{5}{*}{ 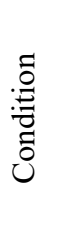 } & 1 & 8.18 & 7.09 & 8.18 & 8.36 & 7.73 & 7.91 & 7.73 & 7.36 & 7.82 \\
\hline & 2 & 7.45 & 6.91 & 7.18 & 5.45 & 7.09 & 5.18 & 6.82 & 5.00 & 6.39 \\
\hline & 3 & 6.55 & 6.18 & 3.36 & 6.36 & 3.36 & 5.00 & 6.00 & 6.55 & 5.42 \\
\hline & 4 & 3.73 & 4.64 & 2.18 & 2.55 & 1.91 & 2.50 & 5.20 & 3.55 & 3.28 \\
\hline & 5 & 2.36 & 1.73 & 1.18 & 1.18 & 1.55 & 1.27 & 5.09 & 3.00 & 2.17 \\
\hline
\end{tabular}

\subsection{Within-condition variance (generalisability)}

Assuming any influence of thematic processing on the sample was negligible, we now seek to determine whether the pairs in each condition were equally good members of that condition.

One-way ANOVA's with repeated measures are carried out on the similarity responses in each condition in order to determine whether the mean similarity ratings of each pair vary significantly from each other. If the pairs are all equivalently good members of that condition, then their means should not vary significantly from each other. A repeated measures ANOVA with a Greenhouse-Geisser correction determined that the mean similarity ratings for the eight pairs within each condition differed significantly in condition two $(\mathrm{F}(3.952,39.521)=4.836, \mathrm{p}=0.003)$, three $(\mathrm{F}(2.959,26.634)=5.612, \mathrm{p}$ $=0.004)$, four $(\mathrm{F}(2.912,23.297)=5.995, \mathrm{p}=0.004)$ and $5(\mathrm{~F}(2.475,24.749)=18.607, \mathrm{p}<0.001)$, but not in condition one $(\mathrm{F}(3.402,34.017)=1.831, \mathrm{p}=0.154)$.

As the mean similarity of the pairs within conditions two to four varies significantly, this suggests that the concepts in each condition are not equally good members of that condition. This implies issues with either the initial stimuli coding or the assumption that the conditions would produce equivalent similarity results across design tasks. Before discussing potential methodological issues, we examine the participants similarity ratings to identify presently unaccounted for factors which may contribute to their perceptions of similarity.

\subsection{Observations from similarity explanations}

The participants' similarity explanations were analysed. Six key observations were drawn from the explanations. Table 5 shows the numbered observation (bold) with accompanying quotes from the explanations as evidence. Two quotes in the same box refer to two participants commenting on the same concept. Two quotes in separate boxes refer to two participants commenting on two different concepts. The implications of these observations are as follows. Factors [1-3b] indicate that the current alignability variables do not capture all of the factors which participants notice in their judgements. The need / function / means variables do not explicitly capture: [1] user interaction, [2] abstract impressions of the product, or [3] relations between e.g. user and product. The pair of concepts to which the quote in observation 4 is referring can be seen in Figure 4. According to the applied coding scheme, there should 
only be commonality at the need level. However, as the participant noted, they share the commonality that they are variants on tents. This commonality is not captured by the existing coding scheme.

Table 5. Observations and supporting evidence

\begin{tabular}{|c|c|}
\hline \# & Observation and evidence \\
\hline \multirow[t]{2}{*}{1} & For the same concept, explanations can focus on properties of the artefact, or user interaction \\
\hline & $\begin{array}{l}\text { "Similarities in resistance training. One connects under desk for feet while working, the other looks like } \\
\text { it's used during a break on the desk." } \\
\text { "Both require resistance work; however, one targets a specific muscle group, whilst the other attempts to } \\
\text { offer a more general solution which enables working various body parts." }\end{array}$ \\
\hline \multirow[t]{3}{*}{2} & Participants notice factors other than need, function, means, e.g. morality, complexity \\
\hline & "both make seating uncomfortable for user. one less sadistic" \\
\hline & "The pull is a simple pull string, the push is a more complex handle that attaches to the barrel." \\
\hline \multirow[t]{3}{*}{3} & Participants notice the relation between the product and the user, e.g. "instruct", "encourage", \\
\hline & "one is light based the other sound. both instruct. both for workouts" \\
\hline & $\begin{array}{l}\text { "Both encourage exercise but by different workouts and engaging different muscle groups and requiring } \\
\text { different skill sets." }\end{array}$ \\
\hline \multirow[t]{2}{*}{$3 b$} & Relations appear to manifest as featural differences \\
\hline & "Both contain the water in a barrel. Different mechanisms to move the water - push v pull." \\
\hline \multirow[t]{2}{*}{4} & $\begin{array}{l}\text { Participants would reference existing product categories as commonalities e.g. fridge, tent, even } \\
\text { when the functions are coded as different }\end{array}$ \\
\hline & "Both are tents but waste-reducing aspects of the two concepts are entirely different" \\
\hline \multirow[t]{2}{*}{5} & Participants commented on concepts having different numbers of functions \\
\hline & $\begin{array}{l}\text { "Both have exact same function - tracks and displays food date, but one has additional function of } \\
\text { keeping food fresh for longer" }\end{array}$ \\
\hline \multirow[t]{2}{*}{6} & Participants would focus on factors related to broader system contexts \\
\hline & "Concept 1 a service + application based product; Concept 2 is a physical product." \\
\hline
\end{tabular}
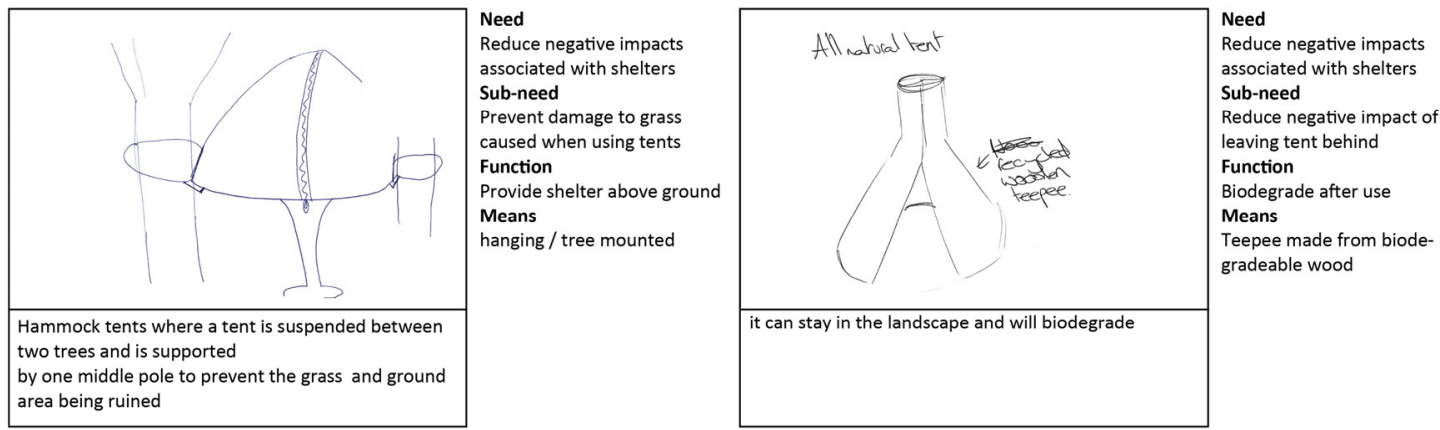

Figure 4. An example of concepts with means-level commonality and function-level differences

Observations [5] and [6] indicate that despite the controls put in place, some concepts were still not homogenous in terms of number of salient functions and exclusion of service elements.

\section{Discussion, limitations, and future work}

\subsection{Thematic processing}

The following discussion assumes that any influence of thematic processing had no meaningful effect on the participants similarity rating. It should be noted however, that interpretation of the similarity explanations should be used as an indicative guide, rather than conclusive method of analysis. Further, thematic processing only occurs with compatible stimuli, i.e. those with potential pre-existing thematic 
relations (Wisniewski and Bassok, 1999). Thus, the lack of thematic processing could be a result of a lack of compatible stimuli in the initial sample. In the future, forced choice tasks could be carried out to determine whether, for example, presumed artefact-artefact affordances contribute to a sense of similarity when compared with pairs with no mutual affordance.

\subsection{Structural alignment processing: Entities and relations}

Defining alignability in terms form of the need, function and means variables, has resulted in generally expected rank ordering of similarity responses across conditions. Owing to high variance within the responses and poor generalisability across conditions this relationship cannot be concluded. Evidence from similarity explanations highlights concept features which contributed to similarity but which have not yet been accounted for. Our alignability definition is centred around describing the design concept; it does not account for the user and the relations between the product and the user. Based on observation 3 and $3 \mathrm{~b}$, participants not only notice relations, but notice them as differences in similarity judgements. Based on the importance of relations (Section 2.1), future alignability schemes need to account for distinct entities and the relations between them.

\subsection{Stimuli creation and methodological limitations}

There were two assumptions behind the stimuli creation method. Firstly, that design concepts can be described in terms of need, function and means variables, and secondly that this could result in unambiguous problem-oriented concept categories. Re-analysis of the concept hierarchies suggests that the initial organisation contained flaws.

\subsubsection{Hierarchical categorisation}

Participants appear to be sensitive to shared similarity to existing concepts (see observation 4). It is unclear as to whether this constitutes a commonality at the need, function or means level. It does however suggest that differentiation of concepts at the superordinate level does not guarantee a lack of commonality at subordinate levels. The created hierarchies were defined around how the concepts addressed the brief. A shared commonality such as 'both are tents' suggests that the participants do not only pay attention to how the concept addresses the brief. Perhaps, to expand on Taura (2016), unambiguous hierarchical classification by any method is not a plausible outcome. While familiar, everyday concepts can be differentiated into categories (Markman and Gentner, 1993), it may not be the case that conceptual design concepts have the same characteristics. Although deign concepts may not be taxonomically organisable, this does not mean that alignability cannot be controlled by variation of commonalities.

\subsubsection{Defining alignability in terms of need, function and means}

Retroactive review of the 'function' levels reveals that despite the function definition provided in Table 2 the function code was applied inconsistently. At least three different types of functions can be identified: (i) what the product does and what it is for ("combust waste for cooking"), (ii) what the product does in terms of a transformation ("condense food waste"), and (iii) functions related to lifecycle ("biodegrade after use"). A more robust theoretical basis for function coding is required.

The high variance in responses could be attributed to an inconsistent hierarchical organisation but may also be a consequence of the granularity of alignability variables. Evidence from the similarity explanations supports this assertion. Participants are seen to refer to phenomena not captured by the alignability scheme, e.g. user movement, impressions of complexity, and the nature of intended behaviour change. Taken together, this suggests that an alignability scheme with improved breadth and granularity may be required.

\subsubsection{Control of extraneous variables}

Despite the inclusion criteria applied, some concepts still contained elements of service systems and participants noticed multiple salient functions for some concepts. While the former issue requires more stringent filtering procedures in future, the latter should be addressed alongside a revision of how 
function is coded in the sample generally. Additional extraneous variables could also be identified among the stimuli, e.g. sketch characteristics such as line type and number of supplementary depictions, or artefact properties such as complexity or number of parts. Controlling for such factors would reduce the potential negative impact of extraneous variables, but may also impair the ecological validity of the sample. If the pairs are over constrained and do not reflect the sort of combinations which occur in practise, then the findings of the research would be of limited practical use.

\subsubsection{Generalisability of similarity across design tasks}

The significant difference in the mean values within each condition cannot be clearly attributed to prominent thematic processing. It is possible that not all pairs may be equally good members of their specified conditions. Inconsistencies in application of alignability codes have already been discussed, however we propose an alternative explanation for lack of generalisability across tasks: differences in 'size' of solution spaces of each design task. The design tasks were assumed to be equivalent in semantic structure and scope. Design task 3 however specifies that solutions must "facilitate transport", no other design task has such a degree of specificity in instruction. This has resulted in a range of solutions constrained around transport methods which results in lower diversity at the 'need' and 'sub need' levels. It is unknown as to whether this could impact the relative similarity between concepts in a solution space.

\subsubsection{Participant demographics and recommendations for future work}

All participants had some experience with the interpretation of design concepts, however the participants varied in terms of their experience and domain background. Future participants will be more homogenous in background and experience, and the equivalence of the design tasks used in the study will be reassessed. The principle requirement for progression of this exploratory study is the development of a new alignability scheme with well-defined constructs at a lower level of granularity than the scheme trialled in this experiment.

\section{Conclusion}

It has been identified that similarity of design concepts may play an important role in various tasks, however little work has been done to integrate knowledge of similarity models from psychology with research in design. Through literature review, insights were gained into two areas, the type of cognitive processing involved in the perception of similarity of concepts generally and the relationship between degree of common features and similarity. Subsequently, further insights were gained through the first experimental study of design concept similarity judgements.

The results of the study show that feature-based similarity judgements predominated in the sample of responses, thus lending support to the possibility that the structural alignment model may be applicable to similarity judgements of design concepts. While no clear evidence of thematic processing was found, a number of ambiguous responses warrant future investigation with different experiment designs. Further, as alignability (defined in terms of common Need, Function and Means variables) decrease, so too did the mean similarity ratings for that condition. However, the relationship between alignability and similarity has not been demonstrated statistically. Through the subsequent discussion, we suggest a number of methodological and conceptual explanations for the high variance and poor generalisability in the responses.

\section{Acknowledgements}

This research was supported by the United Kingdom's Engineering and Physical Sciences Research Council (EPSRC) / University of Strathclyde Research Studentship (CM grant number EP/M506643/1, GC grant number EP/M508159/1); and the EPSRC (AHBD, MG, LH, PC grant number EP/M012123/1).

\section{References}

Bao, Q., Faas, D. and Yang, M. (2018), "Interplay of sketching \& prototyping in early stage product design”, International Journal of Design Creativity and Innovation, pp. 1-23. https://doi.org/10.1080/21650349.2018.1429318 
Bassok, M. and Medin, D.L. (1997), "Birds of a Feather Flock Together: Similarity Judgments with Semantically Rich Stimuli", Journal of Memory and Language, Vol. 36 No. 36, pp. 311-336. https://doi.org/10.1006/jmla.1996.2492

Brown, D.C. (2016), “Observations and Conjectures about Novelty Calculations”, Design Creativity Workshop 2016 / 7th International Conference on Design Computing and Cognition (DCC'16).

Chan, J., Fu, K., Schunn, C., Cagan, J., Wood, K. and Kotovsky, K. (2011), "On the Benefits and Pitfalls of Analogies for Innovative Design: Ideation Performance Based on Analogical Distance, Commonness, and Modality of Examples", Journal of Mechanical Design, Vol. 133 No. 8, pp. 81004. https://doi.org/10.1115/1.4004396

Estes, Z., Golonka, S. and Jones, L.L. (2011), "Thematic Thinking: The Apprehension and Consequences of Thematic Relations", In: Ross, B.H. (Ed.), Psychology of Learning and Motivation, Elsevier, pp. 249-294. https://doi.org/10.1016/B978-0-12-385527-5.00008-5

Fu, K., Chan, J., Cagan, J., Kotovsky, K., Schunn, C. and Wood, K. (2013), "The Meaning of "Near" and "Far": The Impact of Structuring Design Databases and the Effect of Distance of Analogy on Design Output", Journal of Mechanical Design, Vol. 135 No. 2, pp. 21007. https://doi.org/10.1115/1.4023158

Gentner, D. and Markman, A.B. (1995), "Similarity Is Like Analogy: Structural Alignment in Comparison”, In: Cacciari, C. (Ed.), Similarity in Language, Thought and Perception, Brepols, Brussels, pp. 111-147.

Golonka, S. and Estes, Z. (2009), "Thematic relations affect similarity via commonalities", Journal of Experimental Psychology: Learning, Memory, and Cognition, Vol. 35 No. 6, pp. 1454-1464. https://doi.org/10.1037/a0017397

Hay, L., Duffy, A.H.B., McTeague, C., Pidgeon, L. M., Vuletic, T. and Grealy, M. (2017), "Towards a shared ontology: A generic classification of cognitive processes in conceptual design", Design Science, Vol. 3 No. e7. https://doi.org/10.1017/dsj.2017.6

Jang, S. (2014), "The Effect of Image Stimulus on Conceptual Combination in the Design Idea Generation Process", Archives of Design Research, Vol. 27 No. 4, pp. 59-73.

Lin, E.L. and Murphy, G.L. (2001), "Thematic relations in adults' concepts", Journal of Experimental Psychologygeneral, Vol. 130 No. 1, pp. 3-28. https://doi.org/10.1037//0096-3445.130.1.3

Maier, J.R.A. and Fadel, G.M. (2003), “Affordance-Based Methods for Design”, Research in Engineering Design, Vol. 20, pp. 225-239.

Markman, A.B. and Gentner, D. (1993), "Splitting the differences: A structural alignment view of similarity", Journal of Memory and Language, Vol. 32 No. 4, pp. 517-535. https://doi.org/10.1006/jmla.1993.1027

Markman, A.B. and Gentner, D. (1996), "Commonalities and differences in similarity comparisons", Memory \& Cognition, Vol. 24 No. 2, pp. 235-249. https://doi.org/10.3758/BF03200884

Markman, A.B. and Wisniewski, E.J. (1997), "Similar and different: The differentiation of basic-level categories", Journal of Experimental Psychology: Learning, Memory, and Cognition, Vol. 23 No. 1, pp. 54-70. https://doi.org/10.1037/0278-7393.23.1.54

McAdams, D.A. and Wood, K.L. (2002), “A Quantitative Similarity Metric for Design-by-Analogy”, Journal of Mechanical Design, Vol. 124 No. 2, pp. 173-182. https://doi.org/10.1115/1.1475317

Nagai, Y., Taura, T. and Mukai, F. (2009), "Concept blending and dissimilarity: factors for creative concept generation process", Design Studies, Vol. 30 No. 6, pp. 648-675. https://doi.org/10.1016/j.destud.2009.05.004

Taura, T. (2016), Creative Design Engineering: Introduction to an Interdisciplinary Approach, Academic Press.

Tversky, A. (1977), "Features of Similarity", American Psychologist, Vol. 69 No. 5, pp. 379-399. https://doi.org/10.1037/h0021465

van Boeijen, A., Daalhuizen, J., Zijlstra, J. and van der Schoor, R. (2014), Delft design guide: Design methods, Bis Publishers.

Wilkenfeld, M.J. and Ward, T.B. (2001), "Similarity and emergence in conceptual combination", Journal of Memory and Language, Vol. 45 No. 1, pp. 21-38. https://doi.org/10.1006/jmla.2000.2772

Wilson, J.O., Rosen, D., Nelson, B.A. and Yen, J. (2010), “The effects of biological examples in idea generation", Design Studies, Vol. 31 No. 2, pp. 169-186. https://doi.org/10.1016/j.destud.2009.10.003

Wisniewski, E.J. and Bassok, M. (1999), "What makes a man similar to a tie? Stimulus compatibility with comparison and integration", Cognitive Psychology, Vol. 39, pp. 208-238. https://doi.org/10.1006/cogp.1999.0723

Chris Patrick McTeague, PhD Student

University of Strathclyde, Design, Manufacture and Engineering Management

3/2, 38, G42 9SE GLASGOW, United Kingdom

Email: christopher.mcteague@strath.ac.uk 